Supporting Information for

\title{
Influence of Molecular Weight on the Solidification of a Semiconducting Polymer during Time- Controlled Spin-Coating
}

Jin Yeong Na, ${ }^{a}$ Boseok Kang, ${ }^{*, b}$ and Yeong Don Park*,a

${ }^{a}$ Department of Energy and Chemical Engineering, Incheon National University, Incheon 22012, Republic of Korea

${ }^{\mathrm{b}}$ SKKU Advanced Institute of Nanotechnology (SAINT) and Department of Nano

Engineering, Sungkyunkwan University (SKKU), Suwon 16419, Republic of Korea

*Corresponding authors: ydpark@inu.ac.kr (ydp) and bskang88@skku.edu (bk) 
(a)

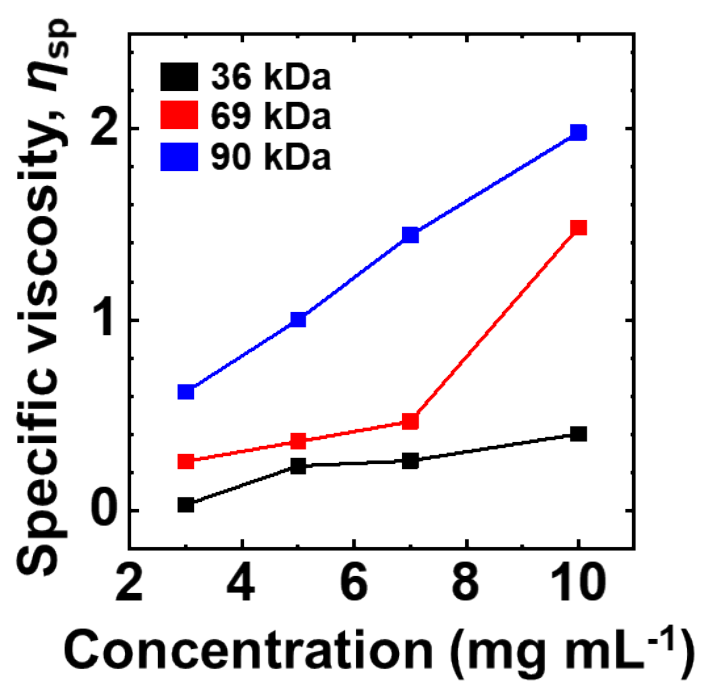

(b)

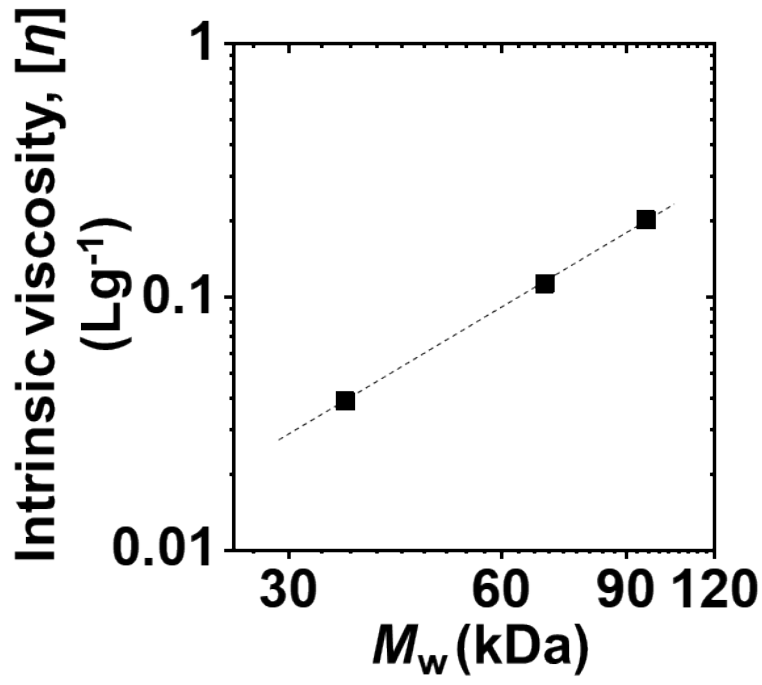

Figure S1. (a) Specific viscosities $\eta_{\mathrm{sp}}$ of the different $M_{\mathrm{w}}$ P3HT solutions as a function of the solution concentration. (b) Intrinsic viscosities $[\eta]$ estimated from Figure S1a. 